\title{
Development of an Ultrasonic Motor Introducing a Curve-shaped Metallic Plate
}

\author{
Kazumi Komatsu $^{1}$, Akihiro Naganawa ${ }^{1, *}$, Yoshinori Ueno $^{1}$, Emilia Abadjieva $^{1}$, Hisashi Ito ${ }^{2}$ \\ ${ }^{1}$ Graduate School of Engineering Science, Akita University, Japan \\ ${ }^{2}$ Best Corporation, Japan
}

Copyright $\bigcirc 2017$ by authors, all rights reserved. Authors agree that this article remains permanently open access under the terms of the Creative Commons Attribution License 4.0 International License

\begin{abstract}
Ultrasonic motors of various drive principles have been developed, but it is difficult to downsized most of them. Hence, an ultrasonic motor which combines a PZT with a metallic plate, which can be downsized easily, is developed by the authors. So far, the studies are focused on the ultrasonic motor with a flat-type metallic plate. In this study, it is tested an ultrasonic motor incorporating a curve-shaped metallic plate, in order to improve performance.
\end{abstract}

Keywords Ultrasonic Motor, Piezoelectric Element, Curve-shaped Metallic Plate, Inchworm-shaped

Deformation, Displacement Expansion

\section{Introduction}

The ultrasonic motors are actuators which have stator that vibrates at an ultrasonic speed and uses friction between the stator and rotor. The first applied in practice ultrasonic motor is a rotational traveling-type, made in Japan in 1986 (Sashida and Kenji [1]). Thereafter, motors of various driving principles are developed (Maeno [2], Pang et al. [3], Liu et al. [4]). They have a lot of features as: high torque at low speed, high torque produced per unit weight, no need of gears and etc. However, most of them are difficult to be downsized and it is not able to incorporate most of the motors into precision devices.

An ultrasonic motor which combines a piezoelectric element with a metallic plate is developed by the authors (Naganawa et al. [5]). Its characteristics are: simple structure, possibility to expand the displacement of the piezoelectric element, easiness to be downsized and etc. So far, the studies are focused on the ultrasonic motor with a flat-type metallic plate.

In this study, an ultrasonic motor having a curve-shaped metallic plate will be introduced. The reason for the improvement of the shape of the metallic plate from flat into curve-shaped, is that the displacement expansion ratio is expected to increase. As a result, it is expected an enhancement of the motor's performance.

\section{Methods}

Structure of the ultrasonic motor consists of stator and rotor, as it is shown in Fig. 1. The stator has a piezoelectric element (lead zirconate titanate, known as "PZT") and a curve-shaped metallic plate in series. When a driving signal is inputted into the piezoelectric element, the voltage causes the piezoelectric element to stretch in longitudinal direction. The extension pushes the metallic plate, which results in bending of the metallic plate in oblique direction. By using the ratio between the stretching displacement of the piezoelectric element and the oblique direction displacement of the metallic plate, it is obtained the extended displacement. It is tested the ratio of the displacement expansion when the radius of the curve-shaped metallic plate is changed from $3 \mathrm{~mm}$ to 20 $\mathrm{mm}$ as shown in Fig. 2 .

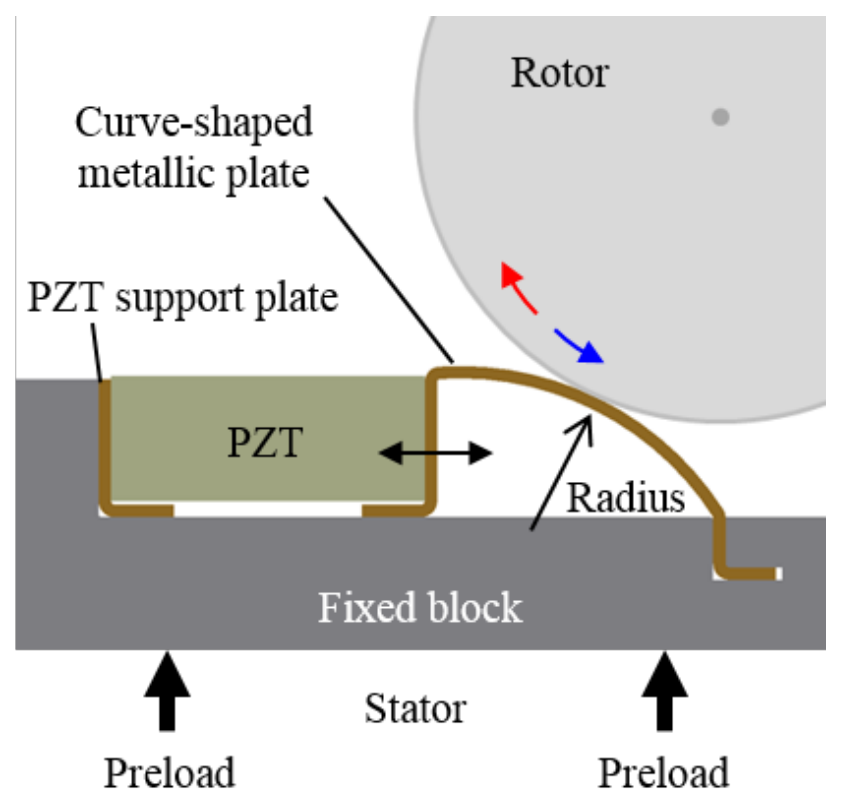

Figure 1. Structure of the ultrasonic motor 


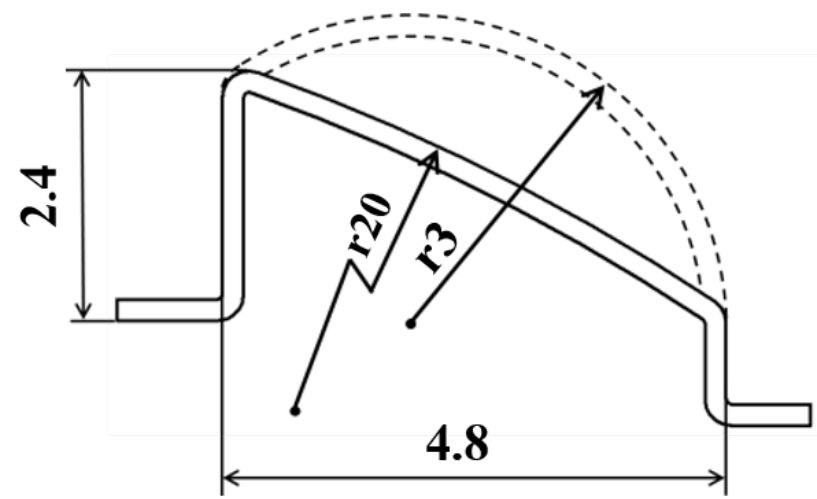

Figure 2. The size of curve-shaped metallic plate in order to calculate the displacement expansion ratio for the radius from $3 \mathrm{~mm}$ to $20 \mathrm{~mm}$

Fig. 3 shows the driving wave. (a) is a wave by the anticlockwise rotation, and (b) is a wave by the clockwise rotation. The wave is a sawtooth-type signal and the ratio of the voltage up and down is $1: 8: 1$. If the rotor rotates in the anticlockwise direction, the wave (which means a slow stretch and rapid shortening) is inputted to the PZT. On the other hand, (b) is a wave to be rotated in the clockwise direction.

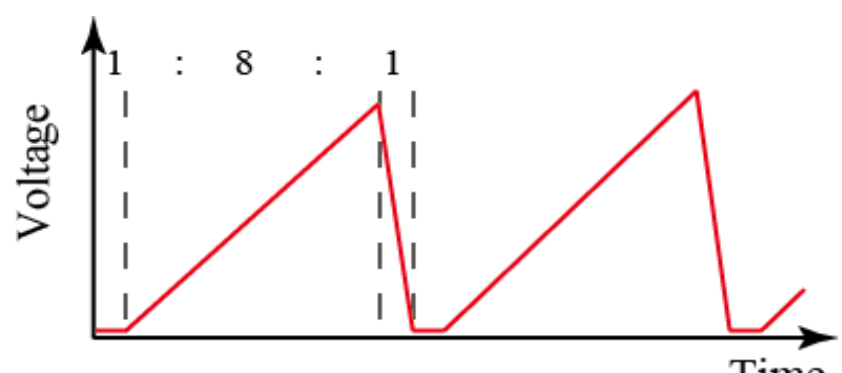

(a) Anticlockwise rotation

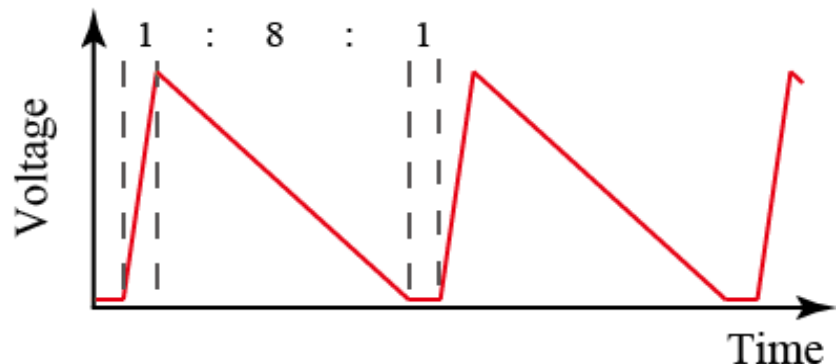

(b) Clockwise rotation

Figure 3. Driving wave.

Fig. 4 shows size of the stator. The height, length and width of the stator of the new ultrasonic motor are $2.4 \mathrm{~mm}$, $10 \mathrm{~mm}$ and $3 \mathrm{~mm}$, respectively. And the PZT is a multilayer-type element which is made by NEC/Tokin Corp. in Japan (type AE0203D04F, length $5 \mathrm{~mm}$, maximum displacement $4.6 \pm 1.5 \mu \mathrm{m} / 150 \mathrm{~V})$. The material of the metallic plate is phosphor bronze with a board thickness of $0.2 \mathrm{~mm}$.

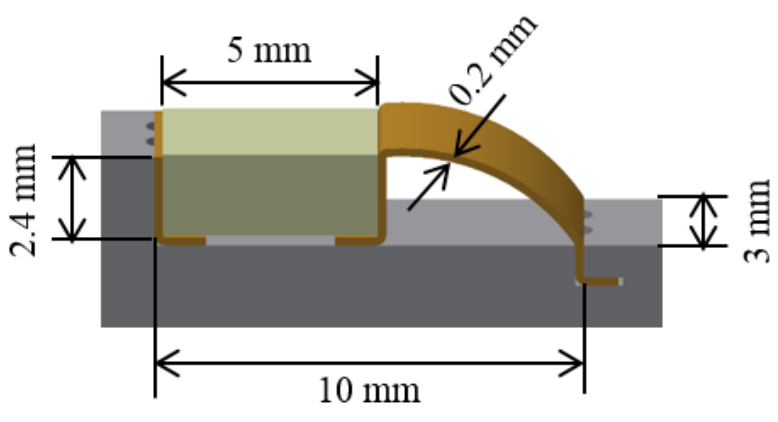

Figure 4. Size of the stator.

Fig. 5 illustrates experimental equipment with the metallic plate with the radius $5 \mathrm{~mm}$ ( $\mathrm{r} 5$ type) for the rotational property. The rotor is made from acrylic and its size is $30 \mathrm{~mm}$ in diameter. The stator is attached to the XY stage and verifies the rotary drive while the position of the stator is adjusted.

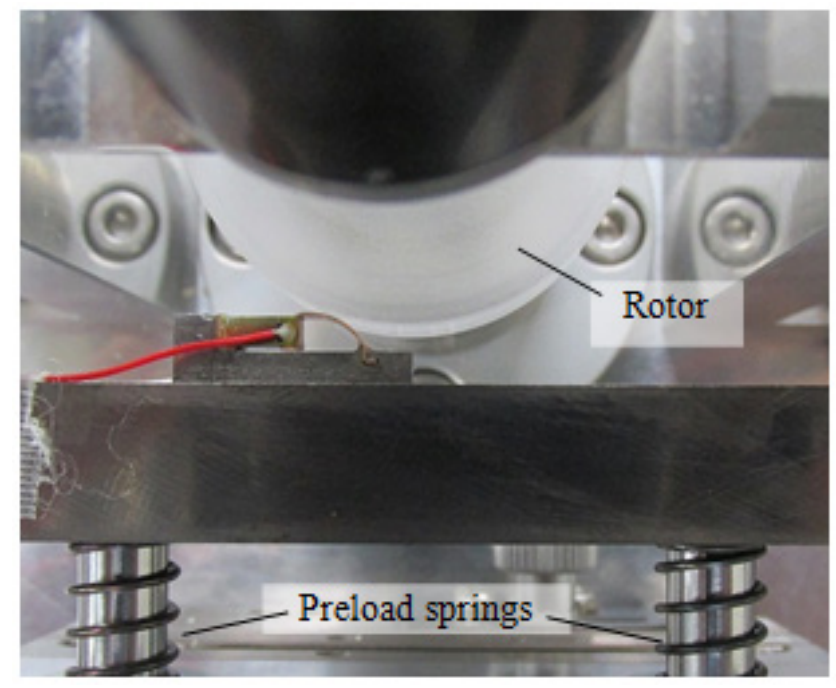

Figure 5. Experimental equipment for the rotational property ( $\mathrm{r} 5$ type)

\section{Results}

Fig. 5 shows the results of the displacement expansion ratio in order to calculate it by the finite element method (FEM). From this figure, it can be seen that the expansion ratio is almost 1 at the radius $4 \mathrm{~mm}$ of the curve-shaped metallic plate and the displacement of the PZT cannot be expanded at the radius $3 \mathrm{~mm}$. On the other hand, as the radius became larger, the expansion ratio increases, and the maximum value is 1.67 times in the horizontal direction and 2.35 in the vertical direction. For this reason, it is decided to compare the performances of the metallic plate with the $r 5$ type and r18 type in this study. 
Fig. 7 and 8 show the results of the FEM analysis. From these results, when the thickness of piezoelectric elements is increased by $3.5 \mu \mathrm{m}$, it can be seen that the curve-shaped metallic plate with the $\mathrm{r} 5$ type and the $\mathrm{r} 18$ type are deformed into an inchworm shape with a maximum displacement of $4.06 \mu \mathrm{m}$ and $5.83 \mu \mathrm{m}$ into the horizontal direction, respectively. Therefore, the ratios of the displacement expansion are 1.16 times and 1.67 times bigger than the displacement of the piezoelectric element, respectively. If it is compared with the flat type metallic plate, the expansion ratio is increased by $36 \%$ for the r 18 type. Moreover, a maximum displacements into the vertical direction are $4.33 \mu \mathrm{m}$ and $8.21 \mu \mathrm{m}$, respectively. Therefore, the ratio of the displacement expansion is 2.35 times for the r18 type.

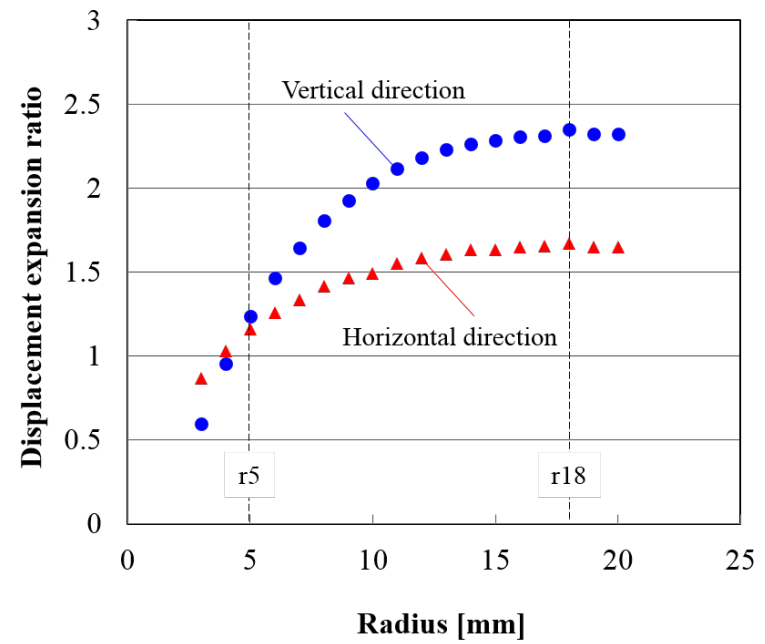

Figure 6. Displacement expansion ratio for the radius of the curved-shaped metallic plate

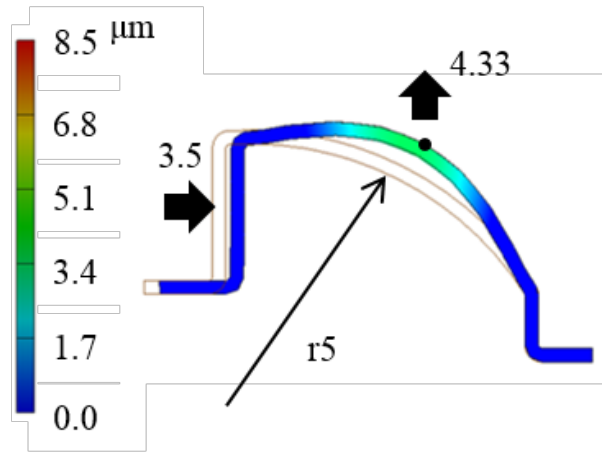

(b) Vertical displacement

(a) Horizontal displacement

Figure 7. Results of FEM analysis ( $r 5$ type)

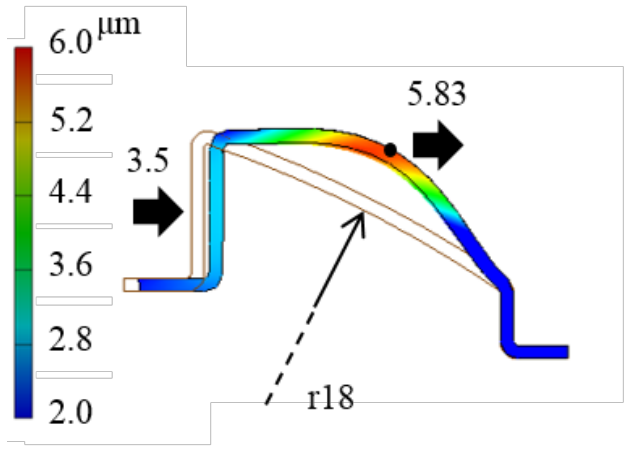

(a) Horizontal displacement

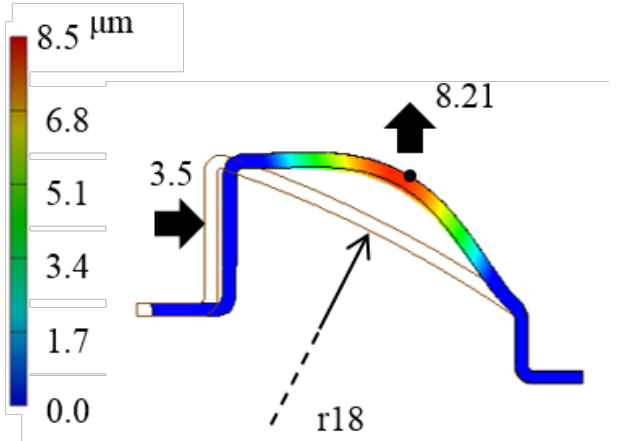

(b) Vertical displacement

Figure 8. Results of FEM analysis (r18 type) 


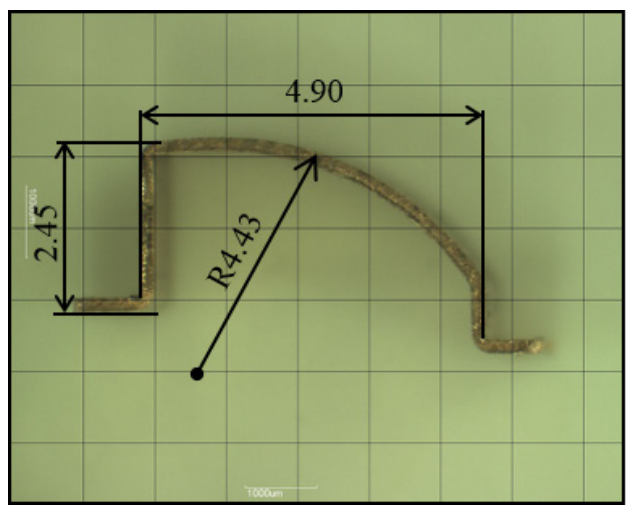

(a) r5 type

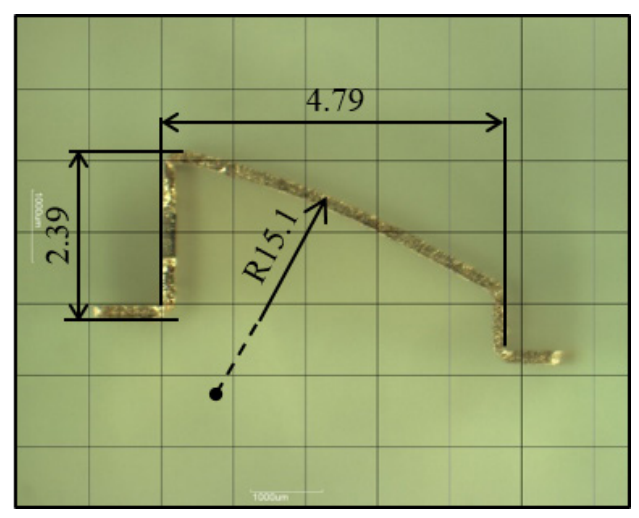

(b) r18 type

Figure 9. Picture of the manufactured curve-shaped metallic plate

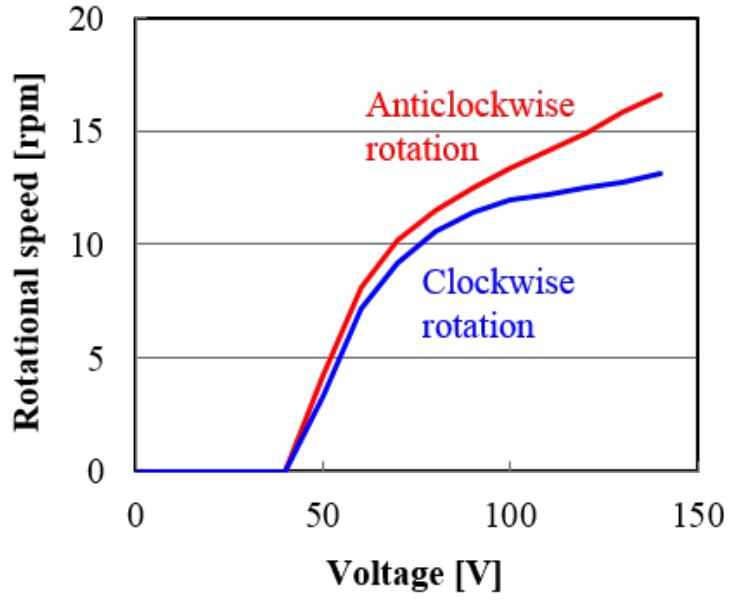

(a) Rotational speed

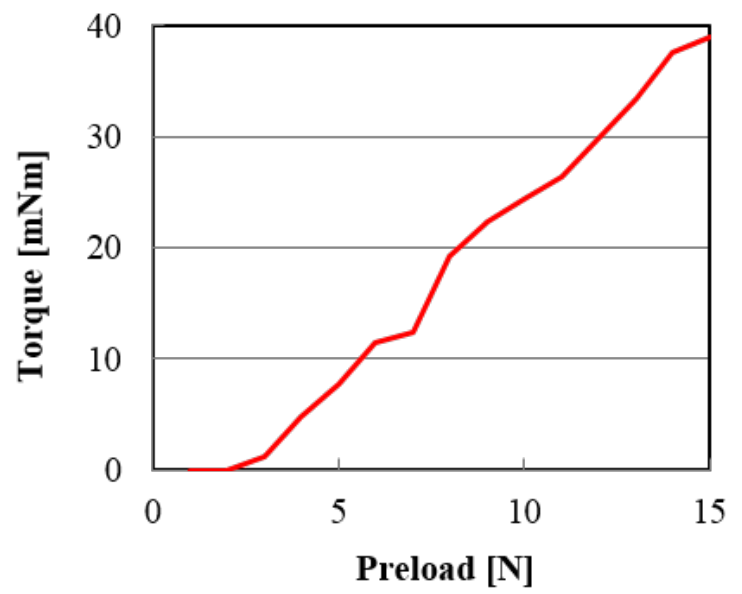

(b) Rotational Torque

Figure 10. Experimental Results ( $r 5$ type).

Fig. 9 shows the picture of the manufactured curve-shaped metallic plate. (a) is the r5 type and (b) is the r18 type. It can be seen that there are some manufactured errors in both curve-shaped metallic plates, because both of them are obtained, while the flat plate is bent.

The rotational property is tested also. Fig. 10 shows the experimental results of the $\mathrm{r} 5$ type metallic plate. (a) is the rotational speed for the applied voltage, and (b) is the rotational torque for the preload. From (a), the maximum rotation speed in the anticlockwise direction is $16.5 \mathrm{rpm}$ when the driving frequency is $12.9 \mathrm{kHz}(140 \mathrm{~V})$ with $11 \mathrm{~N}$ preload. Moreover, the maximum speed in the clockwise direction is $13.1 \mathrm{rpm}$ for the same conditions. On the other hand, from (b), when the preload is bigger, the bigger torque is obtained.

In the future studies, driving performance of r18 type curve-shaped metallic plate will be tested.

\section{Conclusions}

In the study, a new ultrasonic motor structure that utilizes an inchworm-shaped deformation on a curve-shaped metallic plate is offered. The structure consists of a multilayer piezoelectric element and a metallic plate in series. In the finite element analysis, it was confirmed that the expansion ratio can be enhanced by comparing with the conventional flat-type metallic plate. The experimental verification of the motor performance is obtained, while its rotation speed is approximately $15 \mathrm{rpm}$ and it has a torque of $40 \mathrm{mNm}$ for the preload of $15 \mathrm{~N}$.

\section{Acknowledgements}

This work was supported by JSPS KAKENHI Grant Number JP15K05885.

\section{REFERENCES}

[1] Toshiku Sashida, Takashi Kenji (1993) An introduction to ultrasonic motor, Oxford, Oxford University Press. 
[2] Takashi Maeno (2003) Ultrasonic motors. Journal of the Robotics Society of Japan, 21, 10-14 (in Japanese).

[3] Yafei Pang, Ming Yang, Shiyang Li (2012) Rotation angle analyses of plate ultrasonic motor under dual-mode coupling drive, Sensors and Actuators, A173, 202-209.

[4] Yingxiang Liu, Dongmei Xu, Xiaohui Yang, Weishan Chen
(2015) Miniaturized piezoelectric actuator operating in bending hybrid modes, Sensors and Actuators, A235, 158-164.

[5] Akihiro Naganawa, Yoshinori Ueno, Kazumi Komatsu (2016) Development of an ultrasonic motor that uses an inchworm shaped deformation of a metallic plate, World Journal of Engineering and Technology, 4, 100-106. 